\title{
Trauer um Prof. Dr. Wolfgang Edelstein
}

\author{
Engagement für eine demokratische und gerechte Schule \\ $\mathrm{Zu}$ Arbeit und Wirken von Prof. Dr. Wolfgang Edelstein \\ $\left({ }^{\star} 15\right.$. Juni 1929 in Freiburg im Breisgau; $† 29$. Februar 2020 in Berlin)
}

\section{In Memoriam Prof. Dr. Wolfgang Edelstein}

In der pädagogischen Debatte sowohl in der Erziehungswissenschaft als auch in der schulischen Praxis wird das Thema der Demokratie seit den letzten beiden Dekaden intensiv bearbeitet, in jüngerer Zeit mit größerem Nachdruck als je zuvor. Die alte Debatte um die politischen Sozialisationsfunktionen der Schule und um ihre demokratischen Defizite wurde seit etwa dem Jahr 2000 entschieden und neu aufgegriffen. Der etablierten, auf rationales Lernen in homogenen Gruppen zentrierten Schule sollten absichtsvoll und mit großer Professionalität demokratische Umgangs-, Lern- und Lebensformen praxiswirksam entgegengesetzt werden. Den dabei entstandenen und bis heute lebendigen Diskurs hat Wolfgang Edelstein mit großer Entschiedenheit und freundlicher Zuversicht impulsgebend in Gang gesetzt und ausdauernd geführt.

Als erfahrener Lehrer, als Sozial- und Erziehungswissenschaftler und auch als Direktor am Max-Planck-Institut für Bildungsforschung war er in allen Formen der dafür notwendigen Argumentation und Überzeugungsarbeit zuhause. Er sprach viele Menschen in der Schulpädagogik in Wissenschaft und Praxis, in der politischen Bildung und auch in Bildungspolitik und Schulverwaltung an, nahm sie beharrlich und argumentationsstark mit und arbeitete so - mit bis heute anhaltender Wirkung - dafür, dass Pädagogik und Erziehungswissenschaft in den aktuellen Debatten und der praktischen Forschungs- und Entwicklungsarbeit für eine Verbesserung der Schulund Lernqualität um die demokratische Frage nicht mehr herumkommen.

Dabei entstanden - gewissermaßen als Folgewirkung des von ihm gemeinsam mit Peter Fauser gutachterlich begründeten letzten Bund-Länder-Modellprogramms „Demokratie lernen \& leben“ (2001-2007) und danach maßgeblich von Wolfgang Edelsteins Arbeit und Engagement befeuert - der Begriff und ein sowohl theoretisch als auch schulpraktisch belastbares Konzept der „Demokratiepädagogik“. Die dar- 
aus resultierenden Diskurse, Praxisprojekte und Entwicklungsprogramme sind zahlreich und vielschichtig; sie formen die Debatten bis heute. Staatliches Handeln und die Bildungspolitik zeigen sich interessiert - folgen aber noch kaum so prägnant, wie Wolfgang Edelstein und mit ihm die Demokratiepädagogik dies für notwendig hielten und halten.

Die Biographie Wolfgang Edelsteins zeigt, dass Demokratiepädagogik nicht zwingend ein in seinem akademischen Werdegang angelegtes Thema war. Er studierte zunächst Philologie und Linguistik, kam jedoch mit der demokratischen Herausforderung der Institution Schule lebensbiographisch früh und zugleich sehr tief in Berührung: im negativen Sinne in seinen ersten Schuljahren während der NS-Zeit, im positiven Sinne in der auf Anerkennung und sozialen Umgangsformen gründenden Gesamtschulzeit im Exil seiner Jugendjahre in Island.

Sein Weg in der Erziehungswissenschaft führte ihn aus einer frühen, fast zehnjährigen Position als Lehrer und Studienleiter an der Odenwaldschule - die er in den 1950er-Jahren als demokratischen Erfahrungsraum, Ort partizipativer Gestaltung von Lernverhältnissen und professioneller Schulreform erfuhr und mitgestaltete an das Max-Planck-Institut für Bildungsforschung in Berlin, an dessen Gründung er maßgeblich beteiligt war. Als Direktor des Forschungsbereichs „Entwicklung und Sozialisation“ widmete er sich Fragen der kognitiven Psychologie, der sozialen Ungleichheit und der moralischen Entwicklung von Kindern und Jugendlichen sowie einer hiermit korrespondierenden Erziehungspraxis, die ihrerseits bis heute die demokratische Frage in Erziehung und Lernen aus entwicklungstheoretischer Sicht aufwirft.

Wolfgang Edelstein wandte sich insbesondere in der Zeit nach seiner Emeritierung anhaltend, unnachgiebig und mit großer Überzeugungskraft der praktischen Pädagogik zu und setzte sich für eine Schule ein, die nicht nur in ihrer schulgesetzlichen Grundlegung und Verfassung der Demokratie verpflichtet ist, sondern Demokratie als alltägliche Erfahrung, als verantwortetes Lernen und Lehren in einem gerechtigkeitsförderlichen Zusammenwirken von Lehrenden, Lernenden und Eltern ausbuchstabiert, damit Schüler*innen ein „demokratischer Habitus“ zu eigen werden kann. Dieser „demokratische Habitus“ als durch Erfahrung und Handeln der Kinder und Jugendlichen stabil grundiertes Lern- und Bildungsziel ist Kern seines Konzepts von „Demokratiepädagogik“, mit dem - in seinen Worten aus einem Vortrag von 2007 - „Anerkennung und Wertschätzung eines jeden Individuums jederzeit und überall im Gefüge der schulischen Interaktionen“ Ausgangspunkt und Zweck der Schule sein müssen.

Der besondere Verdienst dieser engagierten langjährigen Arbeit kann gar nicht genug gewürdigt werden. Wolfgang Edelstein vermochte mit seiner Überzeugungskraft, seiner präzisen Argumentation und mit seiner freundlichen Beharrlichkeit die Akteure des damaligen Bund-Länder-Programms auch dann zusammenzuhalten, als die- 
sem aufgrund des Kooperationsverbotes von Bund und Ländern im Jahr 2006 seine Anschlussperspektive verlustig ging. Er trug entschieden die dann aufkommende Idee, einen „Dachverband“ der im BLK-Programm engagierten Schulpraktikerinnen, aber auch der damit verbundenen wissenschaftlichen Expert ${ }^{*}$ innen und politischen Akteure zu organisieren, und führte sie weiter. Bei einer „Halbzeitkonferenz" des BLKProgramms 2005 in Magdeburg formulierte er das dort entstandene, knappe und präzise Konsenspapier des „Magdeburger Manifestes“ zusammen mit Gerhard de Hahn und Peter Fauser aus, das dann zugleich bei dieser Gelegenheit zu einem Referenztext für die dabei gegründete „Deutsche Gesellschaft für Demokratiepädagogik e. V.“ werden konnte.

Es war naheliegend, dass er die „Deutsche Gesellschaft für Demokratiepädagogik“ (DeGeDe) in den ersten Jahren als Vorsitzender leitete und sie in der pädagogischen Szene sowie in der Welt der Stiftungen, die sich Bildungs- und Schulfragen zuwenden, etablierte. Die gesellschaftliche und politische Anerkennung für Konzepte, Praxisformen und die wissenschaftliche Begründung der Demokratiepädagogik war ihm bis zu seinem Lebensende ein Herzensanliegen. In vielen Vorträgen, in Gremienarbeiten und wissenschaftlichen Debatten, aber auch in seiner späten Publizistik hatte er die Demokratiepädagogik stets im Fokus. Er gehörte zum Kreis der Gründungsherausgeber des seit 2012 etablierten Jahrbuchs für Demokratiepädagogik. Er war, soweit es zeitlich ging, bei den vielen durch die DeGeDe begründeten und durchgeführten „Demokratietagen“ als Gesprächspartner, anregender Impulsgeber und überzeugender Redner präsent. In besonderer Weise engagierte er sich für die Anwendung des „Klassenrats“ als partizipative, regelhafte, gleichwohl freie Form gestaltbarer Partizipation und früh einsetzender Deliberationskultur an allen Schulen. Besonders war ihm auch an der Etablierung eines „Preis[es] für demokratische Schulentwicklung" gelegen - beide Aufgaben integrierte er in den Handlungskern „seiner" DeGeDe.

Mit unterschiedlichen Partnern gelang es ihm zudem, vor rund zehn Jahren einen berufsaufbauenden Masterstudiengang „Demokratiepädagogische Schulentwicklung und soziale Kompetenzen" an der Freien Universität in Berlin einzurichten, der bis heute modellhaft und erfolgreich, aber auch - aus Edelsteins Sicht muss man wohl sagen: bedauerlicherweise - bislang einzigartig im doppelten Wortsinne geblieben ist.

In besonderer Weise jedoch trug er auch dazu bei, die zu Beginn der Diskurse und Projekte der „Demokratiepädagogik“ skeptische bis misstrauische Disziplin der Fachdidaktik politischer Bildung und damit auch deren Zentrierung auf Demokratiefähigkeit durch „Fachunterricht Politik“ mit der Schulpädagogik und dem damit entstehenden Blick auf die Institution als Ganzes zu verbinden. Dies gelang ihm in der Umgebung eines Wissenschaftsdiskurses, der sich seit dem sogenannten „PISA-Schock" vorzugsweise der systembezogen empirischen und evidenzbasierten Schulforschung und Schulentwicklung zugewendet und damit potenziell von der stark 
auf Personen, biographische Entwicklung und unmittelbare Lernerfahrung ausgerichteten Diskussion und Praxis des Demokratielernens im Kontext der Schule als Ganzer eher abgewendet hat. Wolfgang Edelstein ließ sich von der zu Beginn der Arbeit mit dem BLK-Demokratie-Modellprogramm massiv aufflammenden und kontroversen Debatte um politische Bildung versus Demokratiepädagogik nicht anfechten. Er wusste aus tiefer persönlicher Erfahrung, dass für ein erfolgreiches Lernen von Demokratie und Politik beides notwendig ist: einerseits das Wissen um die Demokratie, damit sie als Wert und normativer Kern subjektiver politischer Bildung und Einstellung zu gewinnen ist, und andererseits die Einübung demokratischer Praxis, damit aus dem Wissen Handlungsfähigkeit, also „demokratische Handlungskompetenz “ werden kann, die sich überall im Lernen und Verhalten und nicht alleine in einem speziellen Unterrichtsfach entfalten muss. In dieser kontinuierlichen Arbeit an einer rationalen Verständigung und begriffsfundierten Überzeugung scheinbar unversöhnlicher Konzepte und Einflussfelder im pädagogischen Diskurs zwischen Schulpädagogik, Bildungsforschung und Didaktik der politischen Bildung liegt sein eigentliches Verdienst.

Das Ziel einer „demokratischen Schule“, die nicht nur Partizipation ermöglichen, Anerkennung stiften sowie die Individualität der Kinder und Jugendlichen respektieren und fördern muss, sondern zugleich an Formen und Sphären der Gerechtigkeit mit professioneller Grundierung zu arbeiten hat, konnte und wollte er nie aus den Augen verlieren. Eine Anerkennung, Kompetenz und Autonomie fördernde Schule, die das Gewicht sozialer Eingebundenheit und deliberativer Verhältnisse für das Lernen grundlegend und in allen Fächern und Domänen ausbuchstabiert - das war das politische und pädagogische Ziel von Wolfgang Edelsteins Arbeit und Engagement: Es ging ihm dabei um eine demokratiepädagogisch fundierte Gesamtschule, die nicht ein parteiliches und ideologisierbares Konstrukt sein sollte, sondern eine der Humanitas verpflichtete Lern- und Lebenswelt in pädagogischer Perspektive. Er blieb daher zeitlebens entschiedener Kritiker des gegliederten Schulsystems, das für ihn in einem unauflöslichen Widerspruch zu den Erfordernissen einer auf das demokratische Zusammenleben und -lernen aller Kinder und Jugendlichen gerichteten Bildung in Chancengerechtigkeit stand.

Zahlreiche Freund ${ }^{\star}$ innen und Kolleg*innen in der praktischen und der wissenschaftlichen Pädagogik teilen inzwischen diese Überzeugung, nicht zuletzt aus der erfahrenen kooperativen und stets bereichernden Zusammenarbeit mit Wolfgang Edelstein.

\section{Wolfgang Beutel}

https://doi.org/10.31244/dds.2020.02.01 